\title{
Evaluating Treatment Strategies in Patients with Early Rheumatoid Arthritis: Merging Modeling Theory with Clinical Practice
}
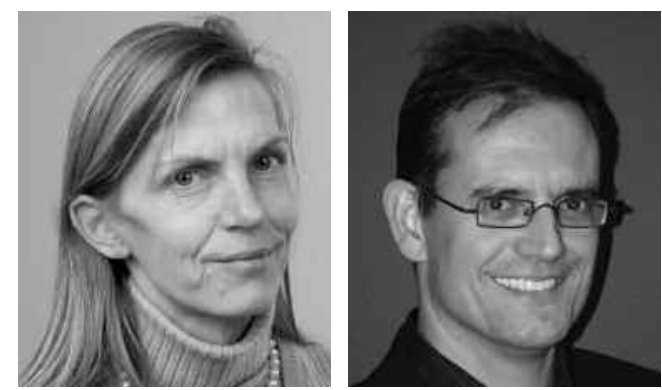

It is impossible to ignore: costs and budget considerations increasingly influence the way rheumatologists can treat and care for their patients in clinical practice. This is especially the case for biologics in the treatment of patients with inflammatory rheumatologic diseases. Until 15 years ago, drug reimbursement decisions in rheumatology were mainly influenced by data on effectiveness and safety. Costs and pricing were a minor issue. This changed somewhat when cyclooxygenase II selective nonsteroidal antiinflammatory drugs were marketed. However, with the introduction of the biologic disease-modifying antirheumatic drugs (DMARD) in rheumatology, the balance between clinical effectiveness and economic aspects of decision options, assessed using cost-effectiveness analyses, played an essential role in reimbursement decisions. This resulted in mounting debates among rheumatologists, and many became involved in negotiations with their hospitals or health insurance companies on how to afford treatments with these drugs within existing budgets. Cost and cost-effectiveness considerations are nowadays also taken into account in treatment recommendations. In the American College of Rheumatology (ACR) recommendations of 2008 on the use of biologic and nonbiologic DMARD in rheumatoid arthritis (RA), the "role of costs for treatment with biologic DMARD" was one of the 5 domains of the literature search that addressed the scientific evidence $^{1}$. Along the same lines, the role of biologics in the European League Against Rheumatism (EULAR) recommendations for the management of RA with synthetic and biological DMARD ${ }^{2}$ is supported by a literature overview on cost-effectiveness of biologics, published separately ${ }^{3}$.

While the clinical position of biologic DMARD in early DMARD-naive patients in terms of effectiveness, safety, and preferences remains an issue for further studies, the above mentioned EULAR overview ${ }^{3}$, as well as a more recent systematic review on cost-effectiveness of biologic DMARD in $\mathrm{RA}^{4}$, conclude that it is unlikely that these drugs will be cost-effective as first-line treatment for early
RA. Similarly, most national reimbursement schedules do not allow biologics as first-line treatment. In this issue of The Journal, Tosh, et al analyzed the cost-effectiveness of different nonbiological treatment strategies in early DMARD-naive patients with RA assuming that patients would continue with a biologic, if they failed 2 classic DMARD according to the British Society of Rheumatology treatment guidelines ${ }^{5}$. Thus, the issue is relevant indeed. While the differences in medication costs between the different nonbiologic strategies that are considered are likely small, large differences in the lifetime costs of RA can be expected when some of these initial strategies allow postponing or reducing the need to initiate biologic DMARD. As such, this analysis contributes insight into the efficiency of different treatment strategies in patients with early RA and eventually will help us understand how this can influence the societal budget.

Of the 6 possible treatment pathways for early RA that were distinguished, this lifetime model showed that, compared to sequential monotherapy, the step-down combination treatment, which comprises initial parallel combination followed by downward dose titration and withdrawal, was the most cost-effective treatment strategy, followed by the intensive step-up combination strategy, comprising parallel combination but with a rapid dose increase of one of the components in case of inadequate response. The step-down combination had a somewhat more favorable incremental costeffectiveness ratio than the intensive step-up combination treatment and dominated (i.e., was much more cost-effective than) the remaining strategies. The net benefit for the UK National Health Service at an acceptability threshold of $£ 20,000$ for one quality-adjusted life-year (QALY) was again highest for the step-down combination and the intensive combination compared to all other strategies.

While the Tosh article can definitely add to the discussion on recommendations for treatment of patients with early RA, it also opens the discussion on some method-

See Cost-effectiveness of combination nonbiologic DMARD strategies in early RA, page 1593 
ological issues. Although cost-effectiveness analyses aim to model real life, the structure of the present model (as most economic models in RA) does not represent what is happening in clinical practice or how rheumatologists treat their patients. For example, evaluation of effectiveness is based on ACR response, while in the majority of countries the recommendations on continuation of treatment are based on changes in disease activity and are operationalized by the Disease Activity Score (DAS) improvement and EULAR response. These measures reflect better how rheumatologists follow their patients in clinical practice and make their treatment decisions. This choice of evaluating initial response by ACR criteria in the article by Tosh, et al was especially surprising because in the model into which patients proceed when they start a biologic, if they failed 2 nonbiologic DMARD, EULAR response is used to evaluate the initial effect of switching to a biologic. As a consequence, by combining 2 models with a different conceptual approach, the virtual patients have different approaches to evaluate effectiveness: the ACR versus the EULAR response ${ }^{6}$. Moreover, the ACR response does not seem to be an obvious measure for defining health states in a cost-effectiveness model, since it is a relative measure, creating difficulties when estimating absolute changes in health-related quality of life, costs, and mortality risk ${ }^{7}$. Of course, the choice can be defended, since the clinical trials that are the basis for the effectiveness data of the different strategies commonly report ACR response as primary outcome. Similar considerations hold true for the use of the Health Assessment Questionnaire (HAQ) as the health component to model the course of disease over time. While the HAQ clearly has a stronger association with longterm costs of RA than the DAS, treatment decisions in daily practice are mainly based on disease activity. Moreover, disease activity provides independent information on the patients' health, costs, and QALY. Technically, the sensitivity of health states may be improved by combining the HAQ with measures such as the DAS ${ }^{7,8}$. Also, modern modeling tools can easily be programmed to link the DAS to HAQ at any point in the course of disease, taking into account the disease duration, level course of DAS, and baseline HAQ and other variables if necessary.

Another issue that deserves reflection is that the choice of treatment strategies in patients with early RA often takes into account the level of disease activity at the start, the presence or absence of factors of poor prognosis, and even preferences of patients. Numerous studies suggest that there is a need to treat patients with poor prognosis more intensively and this is likely what clinicians do in clinical practice $^{9}$. Also, patients that have already failed one biologic are likely to respond differently to the next biologic and those that have had an adverse event are likely to have a lower chance to be treated with another biological.

Until now, the possibility of taking a large number of patient attributes (age, disease duration, level of disease activity, previous failed drugs, experience of adverse events) into account when modeling costs and outcomes in RA has not yet been fully explored in modeling, but definitely needs consideration in the future. This complex variability between patients is not explored in the work presented by Tosh, et al, illustrated by the fact that only 100 individual patients needed to be simulated to receive stable model results. Of course, a comprehensive approach would increase the complexity of models, but new techniques such as discrete event simulation (DES) allow taking into account a set of attributes of the patients that can change over time ${ }^{10}$.

In contrast to generally accepted Markov models, DES is not based on redistribution of patients over health states after fixed episodes of time; it models changes in health based on (1) a set of specific attributes of patients and (2) time to an event that is likely to change the course of health or the patient's attributes. As such, these simulations have high face validity because they represent what happens in daily clinical practice ${ }^{10}$. Clearly, more realistic models require more data, but the rheumatology community has the advantage of worldwide high quality registers that are continuously improved in order to collect data relevant to understanding longterm outcome, and therefore useful for modeling ${ }^{11}$. Of course, these models need extensive validation and, obviously, documentation, which is currently made possible by journals that make appendices available on the $\mathrm{Web}^{10,12,13,14,15,16}$

Cost considerations undeniably influence our daily clinical practice. The present analyses show that in an environment where biologics are available after failure of DMARD, the choice of the initial nonbiological DMARD strategies has important cost-effectiveness implications. This will help in recommendations on efficient treatment of early RA. While cost-effectiveness models aim to reflect real life, it is likely time to adjust the structures of present models so that they simulate better what is happening in clinical practice and acknowledge variability and heterogeneity in a patient population. In this regard, ACR response and HAQ progression are not likely candidates, whereas EULAR response and DAS levels will drive clinical decisions, in addition to a series of personal patient attributes such as a number of prognostic factors, patient's medication history, and preference. With the increasing number of data that become available from both trials and observational studies such as registers, this becomes a feasible goal. As such this invites cost-effectiveness modelers and clinicians to collaborate closely and merge modeling theory with clinical practice.

ANNELIES BOONEN, MD, PhD, Department of Internal Medicine, Division of Rheumatology, Maastricht University Medical Center and Caphri Research Institute, Maastricht; 
JOHAN L. SEVERENS, MSc, PhD, Professor of Health Technology Assessment, Institute of Health Policy and Management, Erasmus University of Rotterdam,

Rotterdam, The Netherlands

Address correspondence to Dr.Boonen; E-mail: a.boonen@mumc.nl

\section{REFERENCES}

1. Saag KG, Teng GG, Patkar NM, Anuntiyo J, Finney C, Curtis JR, et al. American College of Rheumatology 2008 recommendations for the use of nonbiologic and biologic disease-modifying antirheumatic drugs in rheumatoid arthritis. Arthritis Rheum 2008;59:762-84.

2. Smolen JS, Landewe R, Breedveld FC, Dougados M, Emery P, Gaujoux-Viala C, et al. EULAR recommendations for the management of rheumatoid arthritis with synthetic and biological disease-modifying antirheumatic drugs. Ann Rheum Dis 2010;69:964-75.

3. Schoels M, Wong J, Scott DL, Zink A, Richards P, Landewe R, et al. Economic aspects of treatment options in rheumatoid arthritis: a systematic literature review informing the EULAR recommendations for the management of rheumatoid arthritis. Ann Rheum Dis 2010;69:995-1003.

4. Van der Velde G, Pham PA, Machade MAR, Ieraci L, Witteman W, Bombardier C, et al. Cost-effectiveness of biologic response modifiers compared to disease-modifying antirheumatic drugs for rheumatoid arthritis: a systematic review. Arthritis Care Res 2011;63:65-78.

5. Tosh JC, Wailoo AJ, Scott DL, Deighton CM. Cost-effectiveness of combination nonbiologic disease-modifying antirheumatic drug strategies in patients with early rheumatoid arthritis. J Rheumatol 2011;38:1593-600.

6. Brennan A, Bansback N, Nixon R, Madan J, Harrison M, Watson $\mathrm{K}$, et al. Modelling the cost effectiveness of TNF-alpha antagonists in the management of rheumatoid arthritis: results from the British Society for Rheumatology Biologics Registry. Rheumatology 2007;46:1345-54.

7. Bansback N, Ara R, Karnon J, Anis A. Economic evaluations in rheumatoid arthritis: a critical review of measures used to define health states. Pharmacoeconomics 2008;26:395-408.
9. Welsing PM, Severens JL, Hartman M, van Gestel AM, van Riel PL, Laan RF. The initial validation of a Markov model for the economic evaluation of (new) treatments for rheumatoid arthritis. Pharmacoeconomics 2006;24:1011-20.

9. Schoels M, Knevel R, Aletaha D, Bijlsma JW, Breedveld FC, Boumpas DT, et al. Evidence for treating rheumatoid arthritis to target: results of a systematic literature search. Ann Rheum Dis 2010;69:638-43.

10. Caro JJ, Moller J, Getsios D. Discrete event simulation: the preferred technique for health economic evaluations? Value Health 2010;13:1056-60

11. Van Vollenhoven RF, Severens JL. Observational studies: a valuable source for data on the true value of RA therapies. Clin Rheumatol 2011;30 Suppl:S19-S24.

12. Van Gestel A, Severens JL, Webers CAB, Beckers HJM, Jansonius NM, Schouten JSAG. Modeling complex treatment strategies: Construction and validation of a discrete event simulation model for glaucoma. Value Health 2010;13:358-67.

13. Barton P, Jobanputra P, Wilson J, Bryan S, Burls A. The use of modelling to evaluate new drugs for patients with a chronic condition: the case of antibodies against tumour necrosis factor in rheumatoid arthritis. Health Technol Assess 2004;8:1-91.

14. Jobanputra P, Barton P, Bryan S, Burls A. The effectiveness of infliximab and etanercept for the treatment of rheumatoid arthritis: a systematic review and economic evaluation. Health Technol Assess 2002;6:1-110.

15. Chen YF, Jobanputra P, Barton P, Jowett S, Bryan S, Clark W, et al. A systematic review of the effectiveness of adalimumab, etanercept and infliximab for the treatment of rheumatoid arthritis in adults and an economic evaluation of their cost-effectiveness. Health Technol Assess 2006;10:1-229.

16. Brennan A, Bansback N, Reynolds A, Conway P. Modelling the cost-effectiveness of etanercept in adults with rheumatoid arthritis in the UK. Rheumatology 2004;43:62-72.

J Rheumatol 2011;38:1538-40; doi:10.3899/jrheum.110790 